\title{
Lúpus Eritematoso Sistêmico e Tuberculose Renal: Descrição de Nove Casos
}

\section{Systemic Lupus Erythematosus and Renal Tuberculosis: Description of Nine Cases}

\author{
Daniela Cabral de Sousa ${ }^{(1)}$, Marta Maria das Chagas Medeiros ${ }^{(2)}$
}

\section{RESUMO}

Objetivo: O presente estudo tem por objetivo principal descrever uma série de nove casos de tuberculose (TB) renal em pacientes portadores de lúpus eritematoso sistêmico (LES) ocorridos em um período de seis anos em um hospital terciário do Nordeste brasileiro. Métodos: Foram identificados nove pacientes portadoras de LES com baciloscopia e/ou cultura de urina positivas para Mycobacterium tuberculosis no período de outubro de 1998 a novembro de 2004, por intermédio dos registros do Serviço de Microbiologia do Hospital Universitário Walter Cantídio. Foram coletados dados demográficos, dados sobre o LES e sobre a TB renal das respectivas pacientes. Resultados: Todas as pacientes eram do sexo feminino, com idade entre 19 e 58 anos. Quanto às características do LES, todas haviam tido nefrite lúpica em algum momento da evolução, das quais três haviam utilizado ciclofosfamida previamente à infecção. A dose média de prednisona antes do diagnóstico de TB renal variou entre 7, 5 e $20 \mathrm{mg} /$ dia. O diagnóstico de TB renal foi feito por meio de cultura positiva em cinco pacientes e por intermédio de baciloscopia apenas em quatro pacientes. As manifestações laboratoriais mais freqüentes foram leucocitúria e hematúria. A recidiva da TB renal ocorreu em quatro pacientes. Conclusão: A ocorrência de TB renal em pacientes com LES deve ser suspeitada na presença de piúria estéril e/ou hematúria persistentes, em especial em populações de países em desenvolvimento.

Palavras-chave: lúpus, tuberculose, renal, extrapulmonar, infecção.

\section{INTRODUÇÃO}

O lúpus eritematoso sistêmico (LES) é uma doença inflamatória crônica auto-imune de caráter multissistêmico. A ocorrência de eventos infecciosos constitui-se num dos maiores desafios no manejo de pacientes com LES e é muitas vezes responsável pela reagudização da doença (flares), além de representar significativa morbimortalidade para esses pacientes.

\begin{abstract}
Objective: The main objective of the present study was to describe a series of nine cases of renal tuberculosis (TB) in patients diagnosed with systemic lupus erythematosus (SLE) over a period of six years at a tertiary-level hospital in Northeastern Brazil. Methods: Nine SLE patients with renal TB confirmed by bacterioscopy $(n=4)$ or urine culture $(n=5)$ positive for M. tuberculosis between October 1998 and November 2004 were sampled from the records of the microbiology department of Hospital Universitário Walter Cantídio. Data were collected regarding demographics, SLE and renal TB. Results: All patients were female, aged between 19 and 58 years and had a history of lupus nephritis. Three had been on cyclophosphamide therapy prior to TB infection. The average dose of prednisone administered before the diagnosis of renal $T B$ ranged between 7.5 and $20 \mathrm{mg} /$ day. The most common laboratory findings were leukocyturia and hematuria. Renal $T B$ recurred in four patients. Conclusion: The occurrence of renal TB in SLE patients should be suspected in the presence of persistent sterile pyuria and/or hematuria, especially in developing countries.
\end{abstract}

Keywords: lupus, tuberculosis, renal, extra-pulmonary, infection.

A alta freqüência de infecções nos pacientes portadores de LES provavelmente deve-se à combinação de múltiplos distúrbios no sistema imunológico inerentes à doença (neutropenia/ linfopenia, defeitos na quimiotaxia, distúrbios do sistema reticuloendotelial, deficiência do complemento) ${ }^{(1)} \mathrm{e}, \mathrm{em}$ alguns pacientes, deficiência da lecitina ligada à manose ${ }^{(2)}$, bem como associada ao uso de agentes imunossupressores, particularmente corticosteróides em altas doses ${ }^{(1,3)}$.

Recebido em 14/7/2007. Aprovado, após revisão, em 31/10/2007. Declaramos a inexistência de conflitos de interesse

Serviço de Reumatologia do Hospital Universitário Walter Cantídio da Universidade Federal do Ceará (HUWC/UFC).

1. Médica-assistente do Serviço de Reumatologia do HUWC/UFC e membro do Projeto Pronuclear da Sociedade Brasileira de Reumatologia.

2. Professora adjunta da Faculdade de Medicina UFC

Endereço para correspondência: Daniela Cabral de Sousa, Av. Senador Virgílio Távora, 1.857, ap. 402, CEP 60170-251, Fortaleza, CE, e-mail: danicsousa_ce@yahoo.com.br 
Apesar de a maioria dos casos de infecção em pacientes com LES ser atribuída a agentes patogênicos comuns, como vírus e bactérias Gram positivas e negativas, as infecções oportunistas apresentam grande relevância, principalmente levando-se em conta sua dificuldade diagnóstica e a freqüente confusão de seus sintomas com a atividade do $\operatorname{LES}^{(1,3)}$.

As infecções causadas pelo Mycobacterium tuberculosis constituem-se em um problema da saúde pública nos países em desenvolvimento, mesmo em pacientes que não apresentam infecção pelo vírus da imunodeficiência humana (HIV). O risco de tuberculose parece estar aumentado em pacientes com doenças reumáticas crônicas, como o LES, e em pacientes em terapia prolongada com corticosteróides ou imunossupressores ${ }^{(3)}$. A tuberculose (TB), por outro lado, pode mimetizar algumas manifestações clínicas do LES, como artralgias, febre, perda ponderal e indução à formação de auto-anticorpos. Há vários relatos sobre a prevalência de tuberculose em pacientes com LES, com freqüências variáveis e dados particularmente escassos sobre a ocorrência da TB extrapulmonar ${ }^{(3,4,5)}$. A TB renal, além de pouco relatada em associação com o LES, representa um desafio diagnóstico e terapêutico, pois suas manifestações clínicas e laboratoriais confundem-se com a atividade da nefrite lúpica. Enquanto a nefrite ativa pode manifestar-se pela presença de proteinúria, hematúria, cilindrúria e/ou leucocitúria, a TB renal manifesta-se, principalmente, pela presença de hematúria e leucocitúria (piúria estéril). Um correto diagnóstico é de extrema importância, tendo em vista a necessidade de uso de doses altas de corticóide (com ou sem imunossupressor) na nefrite em atividade e de drogas tuberculostáticas, no caso do diagnóstico de infecção pelo Mycobacterium tuberculosis. A não identificação do bacilo como causador das alterações urinárias poderá levar ao uso inapropriado de drogas imunossupressoras que, por sua vez, poderão piorar a TB.

Diante da relativa escassez de relatos sobre TB renal em pacientes com LES, os autores propõem-se a descrever uma série de casos ocorridos num período de seis anos em um serviço terciário de reumatologia.

\section{JUSTIFICATIVA}

A TB renal, por implicar morbimortalidade aos pacientes com LES e por sua semelhança clínica com as manifestações da nefrite lúpica, necessita de melhor investigação e notificação, em especial em populações nas quais a prevalência de tuberculose é elevada. Os casos descritos de TB renal em pacientes com LES são escassos na literatura.

\section{OBJETIVOS}

- Descrever uma série de casos de TB renal em pacientes com LES seguidos em um serviço terciário de reumatologia num período de seis anos.

- Descrever as características clínico-laboratoriais do LES e o uso de corticóide/imunossupressores nos referidos casos.

\section{METODOLOGIA}

Serão descritos todos os casos identificados de TB renal em pacientes com LES acompanhados no Hospital Universitário Walter Cantídio/Universidade Federal do Ceará (HUWC/UFC) no período de outubro de 1998 a novembro de 2004. Desde essa data, o serviço de microbiologia passou a não ter mais meio de cultura para isolamento do bacilo (os exames passaram a ser realizados em laboratórios fora do HUWC), deixando, portanto, de apresentar os registros de exames solicitados.

A seleção dos pacientes foi realizada da seguinte forma:

- Por meio dos registros do Serviço de Microbiologia do HUWC/UFC no referido período: foram selecionados todos os casos com baciloscopia e/ou cultura positiva para BAAR na urina.

- Dentre os selecionados, foram identificados aqueles pacientes cujos pedidos de exames foram provenientes dos serviços de reumatologia, nefrologia e clínica médica do HUWC, serviços nos quais existe maior probabilidade de acompanhamento de pacientes com diagnóstico de LES.

- Todos os pacientes identificados com diagnóstico de LES, segundo os critérios do Colégio Americano de Reumatologia ${ }^{(6)}$, tiveram os prontuários revisados. Foram coletados de cada paciente: dados demográficos, características clínico-laboratoriais do LES, tempo de doença, dose média de corticóide à época do diagnóstico de TB renal, uso prévio de imunossupressores, dados sobre nefrite lúpica prévia, esquema terapêutico usado para TB renal e evolução da infecção.

\section{RESULTADOS}

Foram identificadas nove pacientes com diagnóstico de LES e TB renal no referido período, todas do sexo feminino, 
com idade variando entre 19 e 58 anos à época do diagnóstico da infecção. Três pacientes eram procedentes de Fortaleza e as demais, de outras cidades no interior do Estado.

Quanto às manifestações do LES, vale salientar que todas as pacientes apresentaram glomerulonefrite em algum momento da evolução de sua doença, e cinco pacientes tiveram nefrite classe IV diagnosticada por biópsia renal; as demais, nefrite não classificada. Dentre as cinco pacientes com nefrite classe IV, três haviam feito uso de ciclofosfamida antes do diagnóstico de TB renal, uma teve diagnóstico de nefrite simultaneamente à $\mathrm{TB}$ renal e uma paciente evoluiu com nefrite após o tratamento da TB renal. Estes dois últimos casos fizeram uso de azatioprina. As características clínicas das pacientes encontram-se na tabela 1 .

TABELA 1

Características Clínicas dAS NOVE PACIENTES COM LES E TB RENAL

\begin{tabular}{lc}
\hline Características clínicas & $\mathbf{N}^{0}$ de pacientes \\
\hline Artrite & 9 \\
Lesões cutâneas & 6 \\
\hline Fotossensibilidade & 3 \\
\hline Serosite & 3 \\
\hline Úlceras orais & 1 \\
\hline Psicose & 0 \\
Convulsões & 0 \\
\hline Nefrite & 9 \\
\hline Manifestações hematológicas & 6 \\
\hline
\end{tabular}

O diagnóstico de TB renal foi realizado por meio de cultura positiva para BAAR na urina em cinco pacientes e por pesquisa direta positiva em quatro pacientes. $\mathrm{O}$ intervalo de tempo entre o diagnóstico de LES e o diagnóstico da infecção variou de dois anos e nove meses até 17 anos. À época do diagnóstico de TB renal, a leucocitúria esteve presente em oito pacientes, hematúria em sete e febre em quatro. Apenas três pacientes apresentavam queixa de disúria. $\mathrm{O}$ relato de contatos domiciliares com TB prévia em qualquer outro sítio foi omitido na maioria das pacientes; apenas em um caso houve relato de TB pulmonar ocorrida na mãe da paciente. As características clínico-laboratoriais das pacientes relativas à TB renal encontram-se resumidas na tabela 2 .

Todas as pacientes iniciaram tratamento para TB renal com o clássico esquema tríplice: rifampicina, isoniazida e pirazinamida. A duração do tratamento variou de sete a 14 meses, e as intercorrências ocorridas durante o tratamento
TABELA 2

Características CLÍNICO-LABORATORIAIS DA TB RENAL EM NOVE PACIENTES COM LES

\begin{tabular}{lc}
\hline Características clínico-laboratoriais & $\mathbf{N}^{0}$ de pacientes \\
\hline Febre & 4 \\
Lombalgia & 1 \\
\hline Disúria & 3 \\
\hline Leucocitúria & 8 \\
\hline Hematúria & 7 \\
\hline Pesquisa direta de BK positiva & 9 \\
Cultura para BK positiva & 5 \\
\hline
\end{tabular}

foram as seguintes: intolerância gástrica (quatro pacientes), falta de medicação (três pacientes), reação alérgica à medicação (duas pacientes). A recidiva da TB renal póstratamento ocorreu em quatro pacientes, das quais duas apresentaram duas recidivas cada e duas apresentaram uma recidiva cada.

A dose média de corticóide em uso à época do diagnóstico da TB variou de $7,5 \mathrm{mg} /$ dia a $20 \mathrm{mg} /$ dia.

\section{DISCUSSÃO}

O manejo de pacientes com LES e infecção sempre representou um grande desafio. Há inúmeros relatos na literatura sobre o assunto, em sua maioria estudos retrospectivos. Paton publicou em $1997^{(1)}$ um trabalho de revisão sobre LES e infecção, destacando a importante morbimortalidade tanto das infecções por agentes virais e bacterianos comuns quanto o papel das infecções oportunistas. $\mathrm{O}$ autor ressaltou o papel dos corticóides em altas doses como fator de risco significativo para infecção nesses pacientes e observou a dificuldade de diagnóstico e notificação das infecções oportunistas, as quais muitas vezes mimetizam ou ocorrem simultaneamente à atividade do LES.

Dentre as causas de infecção menos comuns em pacientes com LES, a tuberculose (TB) ganha especial destaque em países em desenvolvimento, nos quais a doença é endêmica. Pacientes imunocomprometidos, além da clássica forma pulmonar, têm maior tendência às apresentações mais graves como TB miliar e formas extrapulmonares. Em 1982, Feng e $\operatorname{Tan}^{(7)}$ publicaram um estudo retrospectivo de 311 pacientes com LES seguidos em um serviço em Singapura, dentre os quais 16 haviam tido diagnóstico de TB (prevalência: 5\%), sendo 12 casos de TB pulmonar e quatro de TB extrapulmonar (um de TB pleural, um de TB linfonodal, um de TB articular e um de TB peritoneal). 
Em 1996, Victorio-Navarra et al. ${ }^{(8)}$, revisando 390 casos de LES nas Filipinas, identificaram 54 pacientes com TB, dentre os quais 42 episódios de TB pulmonar e 29 episódios de TB extrapulmonar, sendo dois casos de TB genitourinária. Hernandez-Cruz et al. ${ }^{(9)}$, da Cidade do México, relataram em 1999 uma série de 30 casos de portadores de doenças reumáticas sistêmicas e TB. Havia 13 pacientes com LES, dentre os quais seis desenvolveram TB miliar. No total de casos (incluindo LES e outros diagnósticos como artrite reumatóide, polimiosite), houve 20 casos de TB extrapulmonar, sendo quatro casos de TB renal. Os autores chamam a atenção para a alta proporção de casos de TB extrapulmonar nessa população de pacientes.

Em 2002, dois outros trabalhos foram publicados relacionando TB e LES. Tam et al. ${ }^{(4)}$ revisaram os dados de 526 pacientes com LES seguidos em um hospital em Hong Kong, identificando 57 pacientes com história de TB (prevalência: 10,8\%). Havia 19 casos de TB pulmonar e 38 casos de TB extrapulmonar, dentre os quais oito casos de TB genitourinária. Os autores ressaltam a alta prevalência de TB na população com LES estudada, 15 vezes maior que na população geral local, além de destacarem a alta prevalência de TB extrapulmonar. Ainda nesse estudo, foi encontrada uma associação entre a presença de nefrite lúpica e um risco duas vezes maior de desenvolver TB. Um segundo trabalho, publicado neste mesmo ano por Yun et al. ${ }^{(5)}$, descreveu uma análise retrospectiva de 283 pacientes com LES e 284 pacientes com artrite reumatóide na Coréia. Dentre os pacientes com LES, 15 apresentavam história de TB (prevalência: 5,3\%), sendo cinco casos de TB extrapulmonar (um de TB articular, um de TB óssea, um de TB renal, um de TB laríngea e um de TB pleural). Mais uma vez, é ressaltada a elevada prevalência de TB em LES, particularmente das formas extrapulmonares.

Sayarlioglu et al. ${ }^{(3)}$, em 2004, publicaram um estudo retrospectivo realizado na Turquia revisando 556 pacientes com LES. Identificaram 20 pacientes com história de TB (prevalência: 3,6\%), dentre os quais nove casos de TB extrapulmonar (três de TB vertebral, duas de TB meníngea, quatro de $\mathrm{TB}$ articular e partes moles). Os autores ressaltam que, na literatura, a prevalência de TB em pacientes com LES varia de $3,6 \%$ a $11,6 \%$.

Diante do exposto, podemos observar que a prevalência de TB é bastante variável em pacientes com LES e que a maior parte dos trabalhos sobre o assunto provém de países em que a tuberculose é endêmica. Também constatamos que, apesar da TB extrapulmonar aparentemente ter maior prevalência em pacientes com LES em relação à população geral, os relatos ainda são escassos. Particularmente, a TB renal é pouco citada, o que nos faz questionar se tal fato deve-se à raridade da condição ou à dificuldade diagnóstica e à pouca investigação dos casos.

Por outro lado, deve-se considerar ainda a dificuldade em distinguir os sinais de TB renal de uma possível nefrite lúpica. Em um estudo de 26 pacientes com TB renal realizado na Turquia, publicado em $2005^{(10)}$, as manifestações de sedimento urinário foram hematúria e/ou leucocitúria, presentes em $87 \%$ dos casos. As manifestações clínicas, como lombalgia, disúria, dor suprapúbica, estavam presentes em apenas uma parcela dos pacientes, e a manifestação mais freqüente (disúria) ocorria em apenas $46 \%$ dos casos. Em relação à nefrite lúpica, as manifestações urinárias são variadas, dependendo do tipo histológico. Podem estar presentes hematúria, leucocitúria, cilindrúria e proteinúria. Apesar de esta última ser um marcador importante da nefrite lúpica, não é específica, podendo estar presente em condições como diabetes, hipertensão arterial sistêmica ou doença aterosclerótica ${ }^{(11)}$.

Julgamos, portanto, relevante descrever essa série de casos ocorridos no período de seis anos em um serviço de referência no Nordeste do Brasil, país em que a tuberculose ainda se constitui um problema de saúde pública. Uma limitação de nosso estudo seria a falta de registro do total de pacientes portadores de LES seguidos em nosso serviço no referido período, o que nos possibilitaria ter uma idéia melhor da magnitude do problema. Gostaríamos de destacar o fato de que todas as pacientes com diagnóstico de TB renal também apresentaram nefrite lúpica em algum momento de sua evolução, o que causou dificuldades de ordem diagnóstica e terapêutica. Apesar do pequeno número de casos e do caráter descritivo do presente estudo, chamou-nos atenção a alta freqüência de recidiva da $\mathrm{TB}$ renal na população estudada. Isto pode reforçar a idéia de que o uso de corticóides e/ou imunossupressores, além dos distúrbios imunológicos inerentes ao LES, pode contribuir não só para a aquisição como também para a dificuldade de resolução da infecção nesses pacientes.

Em resumo, esta descrição de uma série de nove casos de TB renal em pacientes com LES tem o propósito de alertar para a ocorrência de tal associação, sugerindo a pesquisa do Mycobacterium tuberculosis em pacientes com LES com piúria estéril e/ou hematúria persistente, em especial em populações de países em desenvolvimento. 


\section{REFERÊNCIAS}

1. Paton NI: Infections in systemic lupus erythematosus patients. An Acad Med Singapore 26: 694-700, 1997.

2. Kang I, Park SH: Infectious complications in SLE after immunosuppressive therapies. Curr Opin Rheumatol 15(5): 528-34, 2003.

3. Sayarlioglu M, Inanc M, Kamali S, et al.: Tuberculosis in Turkish patients with systemic lupus erythematosus: increased frequency of extrapulmonary localization. Lupus 113: 274-8, 2004.

4. Tam LS, Li EK, Wong SM, Szeto CC: Risk factors and clinical features for tuberculosis among patients with systemic lupus erythematosus in Hong Kong. Scand J Reheumatol 31: 296-300, 2002.

5. Yun JE, Lee SW, Kim TH, et al.: The incidence and clinical characteristics of Micobacterium tuberculosis infection among systemic lupus erythematosus and rheumatoid arthritis patients in Korea. Clin Exp Rheumatol 20: 127-32, 2002.
6. Tan EM, Cohen AS, Fries JF, et al.: The 1982 revised criteria for the classification of systemic lupus erythematosus. Arthritis Rheum 25: 1271-7, 1982.

7. Feng PH, Tan TH.: Tuberculosis in patients with systemic lupus erythematosus. Ann Rheum Dis 41: 11-4, 1982.

8. Victorio-Navarra STG, Dy EER, Arroyo CG, Torralba TP: Tuberculosis among Filipino patients with systemic lupus erythematosus. Semin Arthritis Rheum 26(3): 628-34, 1996.

9. Hernandez-Cruz B, Sifuentes-Osornio J, Ponce-de-Leon Rosales S, Ponce-de-Leon Garduno A, Diaz-Jouanen E: Mycobacterium tuberculosis infection in patients with systemic rheumatic diseases. A case series. Clin Exp Rheumatol 17: 289-96, 1999.

10. Altintepe L, Tonbul HZ, Ozbey I, et al.: Urinary tuberculosis: ten years' experience. Ren Fail 27: 657-61, 2005.

11. Dooley MA, Aranow C, Ginzler EM: Review of ACR renal criteria in systemic lupus erythematosus. Lupus 13: 857-60, 2004. 